$\mathrm{J}$ o u r n a l of

Mathematics

and Applications

JMA No 41, pp 171-180 (2018)

\title{
Location of Zeros of Lacunary-type Polynomials
}

\author{
Idrees Qasim, Tawheeda Rasool and Abdul Liman
}

ABstRaCT: In this paper, we present some interesting results concerning the location of zeros of Lacunary-type of polynomial in the complex plane. By relaxing the hypothesis and putting less restrictive conditions on the coefficients of the polynomial, our results generalize and refines some classical results.

AMS Subject Classification: 30A01, 30C10, 30C15.

Keywords and Phrases: Lacunary polynomials; Zeros; Eneström-Kakeya Theorem.

\section{Introduction}

The study of the zeros of a polynomial dates from about the time when the geometric representation of complex numbers was introduced into mathematics. The first contributors of the subject were Guass and Cauchy. Since the days of Gauss and Cauchy many mathematicians have contributed to the further growth of the subject. The classical results of Cauchy [3], concerning the bounds for the moduli of the zeros of a polynomial states

Theorem A. $P(z):=\sum_{j=0}^{n} a_{j} z^{j}$ is a polynomial of degree $n$ and $M=\max _{0 \leq j \leq n-1}\left|\frac{a_{j}}{a_{n}}\right|$, then all the zeros of $P(z)$ lie in

$$
|z| \leq 1+M
$$

There exists several generalizations and improvements of this result (for reference see [12] and [13]). As an improvement of this result, Joyal, Labelle and Rahman [8] 
proved that, if $B=\max _{0 \leq j \leq n-1}\left|a_{j}\right|$, then all the zeros of the polynomial $P(z):=$ $z^{n}+\sum_{j=0}^{n-1} a_{j} z^{j}$ are contained in the circle

$$
|z| \leq \frac{1}{2}\left\{1+\left|a_{n-1}\right|+\left\{\left(1-\left|a_{n-1}\right|\right)^{2}+4 B\right\}^{\frac{1}{2}}\right\} .
$$

Next we state the following elegant result which is commonly known as EneströmKakeya Theorem in the theory of distribution of zeros of polynomials.

Theorem B. Let $P(z):=\sum_{j=0}^{n} a_{j} z^{j}$ be a polynomial of degree $n$ such that

$$
a_{n} \geq a_{n-1} \geq \cdots \geq a_{1} \geq a_{0}>0,
$$

then all the zeros of $P(z)$ lie in $|z| \leq 1$.

Theorem $B$ was proved by Eneström [4], independently by Kakeya [9] and Hurwitz [7]. Applying this result to the polynomial $z^{n} P\left(\frac{1}{z}\right)$, one gets equivalent form of Eneström-Kakeya Theorem which states that

Theorem C. If $P(z):=\sum_{j=0}^{n} a_{j} z^{j}$ is a polynomial of degree $n$ such that

$$
a_{0} \geq a_{1} \geq \cdots \geq a_{n-1} \geq a_{n}>0,
$$

then all the zeros of $P(z)$ lie in $|z| \geq 1$.

Applying the above results to the polynomial $P(t z)$, the following more general result is immediate:

Theorem D. Let $P(z):=\sum_{j=0}^{n} a_{j} z^{j}$ be a polynomial of degree $n$ such that

$$
a_{n} t^{n} \geq a_{n-1} t^{n-1} \geq \cdots \geq a_{1} t \geq a_{0}>0,
$$

then all the zeros of $P(z)$ lie in $|z| \leq t$ and in case

$$
0<a_{n} \leq a_{n-1} t^{n-1} \leq \cdots \leq a_{1} t^{n-1} \leq a_{0} t^{n},
$$

then $P(z)$ has all zeros in $|z| \geq \frac{1}{t}$.

Now consider the class of polynomials

$$
\begin{gathered}
P(z):=a_{0}+a_{1} z+\cdots+a_{\mu} z^{\mu}+a_{n_{1}} z^{n_{1}}+a_{n_{2}} z^{n_{2}}+\cdots+a_{n_{k}} z^{n_{k}}, \\
0<n_{0}=\mu<n_{1}<n_{2}<\cdots<n_{k}, \quad a_{0} a_{\mu} a_{n_{1}} a_{n_{2}} \ldots a_{n_{k}} \neq 0 .
\end{gathered}
$$

Here the coefficients $a_{j}, 0 \leq j \leq \mu$, are fixed, the coefficients $a_{n_{j}}, j=1,2, \ldots, k$ are arbitrary and the remaining coefficients $a_{j}$ are zero.

Landau ([10] and [11]) Initiated the study of polynomials of this form in 1906-7. 
He considered the case $\mu=1, k=1$ or 2 and proved that every trinomial

$$
a_{0}+a_{1} z+a_{n} z^{n}, \quad a_{1} a_{n} \neq 0, \quad n \geq 2
$$

has at least one zero in the circle $|z| \leq 2\left|\frac{a_{0}}{a_{1}}\right|$ and every quadrinomial

$$
a_{0}+a_{1} z+a_{m} z^{m}+a_{n} z^{n}, \quad a_{1} a_{m} a_{n} \neq 0, \quad 2 \leq m<n,
$$

has at least one zero in the circle $|z| \leq \frac{17}{3}\left|\frac{a_{0}}{a_{1}}\right|$. Thus for these cases Landau proved the existence of a circle $|z|=R\left(a_{0}, a_{1}\right)$ containing at least one zero of $P(z)$. He also raised the question as to whether or not a circle with this same property existed in the case $\mu=1$ and $k$ arbitrary.

An affirmative reply was given in 1907 by Allardice [1] who proved that when $\mu=1$, at least one zero of $P(z)$ lies in the circle

$$
|z| \leq\left|\frac{a_{0}}{a_{1}}\right| \prod_{j=1}^{k} \frac{n_{j}}{n_{j}-1}
$$

and by Fejér ([6], [5]) who proved that, when $a_{1}=a_{2}=\cdots=a_{\mu-1}=0$, at least one zero of $P(z)$ lies in the circle

$$
|z| \leq\left\{\left|\frac{a_{0}}{a_{\mu}}\right| \prod_{j=1}^{k} \frac{n_{j}}{n_{j}-\mu}\right\}^{\frac{1}{\mu}} .
$$

Another result which is instructive is the one due to Van Vleck [14], who proved that the polynomial

$$
P(z):=1+a_{r} z^{r}+a_{r+1} z^{r+1}+\cdots+a_{n} z^{n}, \quad r<n, \quad a_{r} \neq 0
$$

has at least $r$ zeros in the disk $|z| \leq\left[\frac{C(n, r)}{\left|a_{r}\right|}\right]^{\frac{1}{r}}$.

Recently Aziz and Rather [2] proved the following result for Lacunary-type of polynomials.

Theorem E. For any given positive number $t$, all the zeros of the polynomial

$$
P(z):=a_{n} z^{n}+a_{\mu} z^{\mu}+a_{\mu-1} z^{\mu-1}+\cdots+a_{1} z+a_{0}, \quad \mu<n, \quad a_{0} a_{\mu} a_{n} \neq 0
$$

lie in the circle

$$
|z| \leq \max \left\{N_{p, t}^{\frac{1}{n-\mu}}, N_{p, t}^{\frac{1}{n}}\right\}
$$

where

$$
N_{p, t}=(\mu+1)^{\frac{1}{q}}\left\{\sum_{j=0}^{\mu}\left|\frac{a_{j}}{a_{n} t^{n-j}}\right|^{p}\right\}^{\frac{1}{p}},
$$


$p>1, q>1$ with $p^{-1}+q^{-1}=1$.

In this paper, we consider the following Lacunary-type of polynomials of type (1.1) with $k=1$ and $\mu$ arbitrary

$$
P(z):=a_{n} z^{n}+a_{\mu} z^{\mu}+a_{\mu-1} z^{\mu-1}+\cdots+a_{1} z+a_{0}, \quad \mu<n, \quad a_{0} a_{\mu} a_{n} \neq 0,
$$

and prove some results concerning the bounds for the zeros of polynomials of this form.

\section{Main Results}

Theorem 1. Let $P(z)$ be a polynomial of type (1.2) which does not vanish in $|z|<t$, where $t>0$, then for $p>0, q>0, p^{-1}+q^{-1}=1$, all the zeros of $P(z)$ lie in

$$
|z-t| \leq A=(\mu+2))^{\frac{1}{q}}\left\{\sum_{j=0}^{\mu+1}\left|\frac{t a_{j}-a_{j-1}}{a_{n} t^{n-j}}\right|^{p}\right\}^{\frac{1}{p}},
$$

where $a_{-1}=a_{\mu+1}=0$.

Theorem 1 states that if no zero of a polynomial $P(z)$ of type (1.2) lie in $|z|<t, t\rangle$ 0 , then all its zeros will lie in the region between the circles $|z|<t$ and $|z-t| \leq A$, where $\mathrm{A}$ is defined above. As an example we take a polynomial $P(z)=z^{3}+2 z+3$. Here $\mu=1$ and $n=3$. We make use of Wolfram Mathematica to visualize the zeros of the above polynomial in a specific region (figure 1). The zeros of the polynomial are $(-1.17951,0) ; \quad(0.589755,-1.74454) ; \quad(0.589755,1.74454)$. Take $t=1$, clearly $P(z)$ does not vanish in $|z|<1$. Therefore it follows from Theorem 1 with $p=2, q=2$ that all the zeros of $P(z)$ lie in $|z-1| \leq 8.48528$.

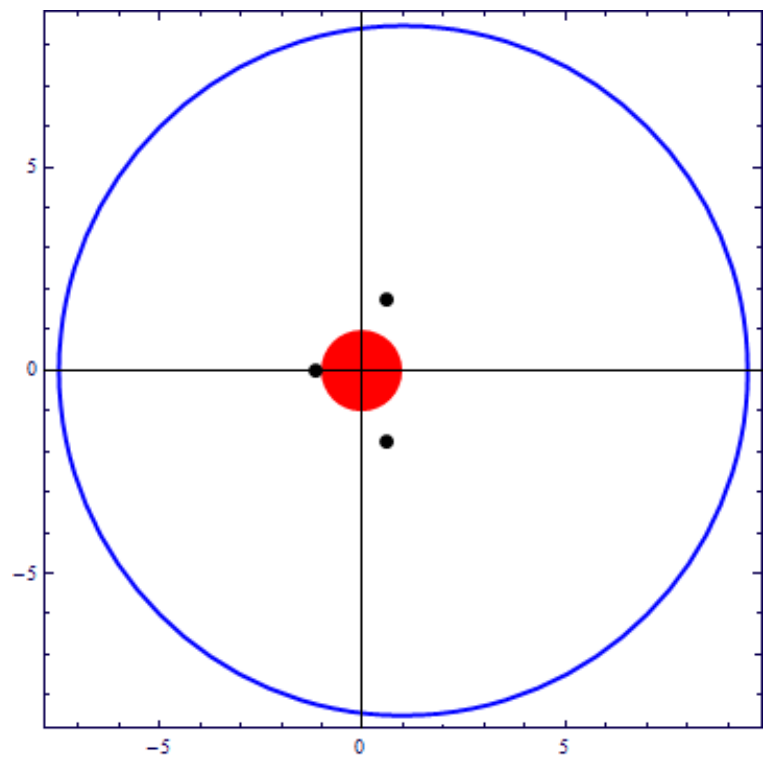


Thus it is clear from figure 1 that all the zeros of the above polynomial lie in the unshaded region between the circles $|z|<1$ and $|z-1| \leq 8.48528$.

For $\mu=n-1$, the Lacunary polynomial $P(z)$ in Theorem 1 reduces to a simple polynomial of degree $n$ and yields the following result.

Corollary 1. Let $P(z):=\sum_{j=0}^{n} a_{j} z^{j}$, be a polynomial of degree $n$ which does not vanish in $|z|<t$ where $t>0$, then for $p>0, q>0, p^{-1}+q^{-1}=1$, all the zeros of $P(z)$ lie in

$$
|z-t| \leq(n+1)^{\frac{1}{q}}\left\{\sum_{j=0}^{n}\left|\frac{t a_{j}-a_{j-1}}{a_{n} t^{n-j}}\right|^{p}\right\}^{\frac{1}{p}}
$$

where $a_{-1}=0$.

As an example, we take $P(z)=2 z^{2}+2 z+3$, having zeros $(-0.5,-1.11803)$; $(-0.5,1.11803)$. Also take $t=1$. Clearly these zeros does not lie in $|z|<1$. Moreover from Corollary 1, it is clear that all the zeros lie in the region between the circles $|z|<1$ and $|z-1|<2.738$ as is clear from figure 2 .

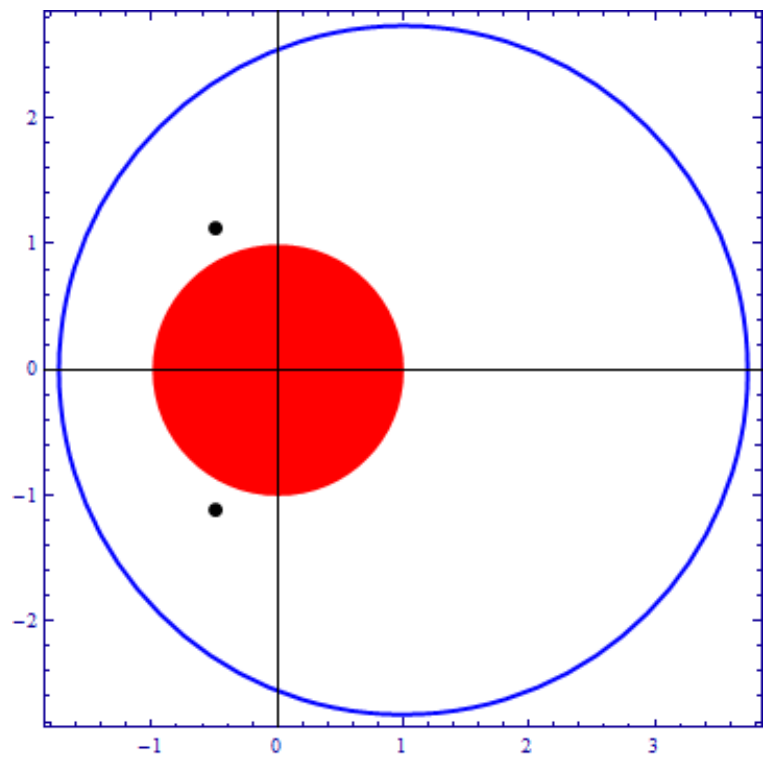

For $\mu=1$, we have the following result for trinomial of degree $n$.

Corollary 2. Let $P(z)=a_{n} z^{n}+a_{1} z+a_{0}$, be a trinomial of degree $n$ which does not vanish in $|z|<t$ where $t>0$, then for $p>0, q>0, p^{-1}+q^{-1}=1$, all the zeros of $P(z)$ lie in

$$
|z-t| \leq 3^{\frac{1}{q}}\left\{\sum_{j=0}^{2}\left|\frac{t a_{j}-a_{j-1}}{a_{n} t^{n-j}}\right|^{p}\right\}^{\frac{1}{p}} .
$$


Letting $q \rightarrow \infty$ so that $p=1$ in Theorem 1 , we get the following result.

Corollary 3. Let $P(z)$ be a polynomial of type (1.2) which does not vanish in $|z|<t$, where $t>0$, then all the zeros of $P(z)$ lie in the circle

$$
|z-t| \leq \sum_{j=0}^{\mu+1}\left|\frac{t a_{j}-a_{j-1}}{a_{n} t^{n-j}}\right| .
$$

In particular for $t=1$, we have the following Corollary by restricting the coefficients of the polynomial.

Corollary 4. Let $P(z)$ be a polynomial of type (1.2) with real coefficients, which does not vanish in $|z|<1$ and

$$
a_{\mu} \geq a_{\mu-1} \geq \cdots \geq a_{1} \geq a_{0}>0, \quad a_{n}>0,
$$

then all the zeros of the polynomial lie in the circle

$$
|z-1| \leq 2 \frac{a_{\mu}}{a_{n}} .
$$

In other words all the zeros of the polynomial $P(z)$ which does not vanish in $|z|<1$ lie in the region

$$
\left\{z: 1 \leq|z| \cap|z-1| \leq 2 \frac{a_{\mu}}{a_{n}}\right\} .
$$

If we reverse the monotonicity of the coefficients of the polynomial, we get the following result.

Corollary 5. Let $P(z)$ be a polynomial of type (1.2) with real coefficients, which does not vanish in $|z|<1$ and

$$
a_{0} \geq a_{1} \geq \cdots \geq a_{\mu}>0, \quad a_{n}>0,
$$

then all the zeros of $P(z)$ lie in the circle

$$
|z-1| \leq 2 \frac{a_{0}}{a_{n}} .
$$

In other words if $P(z)$ does not vanish in $|z|<1$, then all the zeros of $P(z)$ lie in the region

$$
\left\{z: 1 \leq|z| \cap|z-1| \leq 2 \frac{a_{0}}{a_{n}}\right\} .
$$

Again we make use of WOLFRAM MATHEMATICA to show that the bounds obtained in our results are sharper then the prior ones. For this we take the following 
Lacunary polynomial of type (1.2)

$$
P(z):=z^{3}+2 z+12 \text {. }
$$

Its zeros are given below

$(-2),(1-2.23607 \iota),(1+2.23607 \iota)$. The bounds for the zeros of the above Lacunary polynomial $P(z)$ obtained by using different results are given in the following table.

\begin{tabular}{|l|l|l|}
\hline \multicolumn{3}{|c|}{ Bounds for the zeros of $P(z):=2 z^{8}+z+5$ by using different results } \\
\hline S.No. & Theorems & Bounds \\
\hline 1 & Landau's Theorem for Trinomials & $|z| \leq 12$ \\
2 & Theorem A & $|z| \leq 13$ \\
3 & Theorem C & $|z| \geq 1$ \\
4 & Corollary 2 & $1 \leq|z| \cap|z-2| \leq 6.4807$ \\
5 & Corollary 3 & $1 \leq|z| \cap|z-2| \leq 6$ \\
\hline
\end{tabular}

In order to visualize the above bounds we draw the following figure in which circles of different colours represents different bounds obtained by various results.

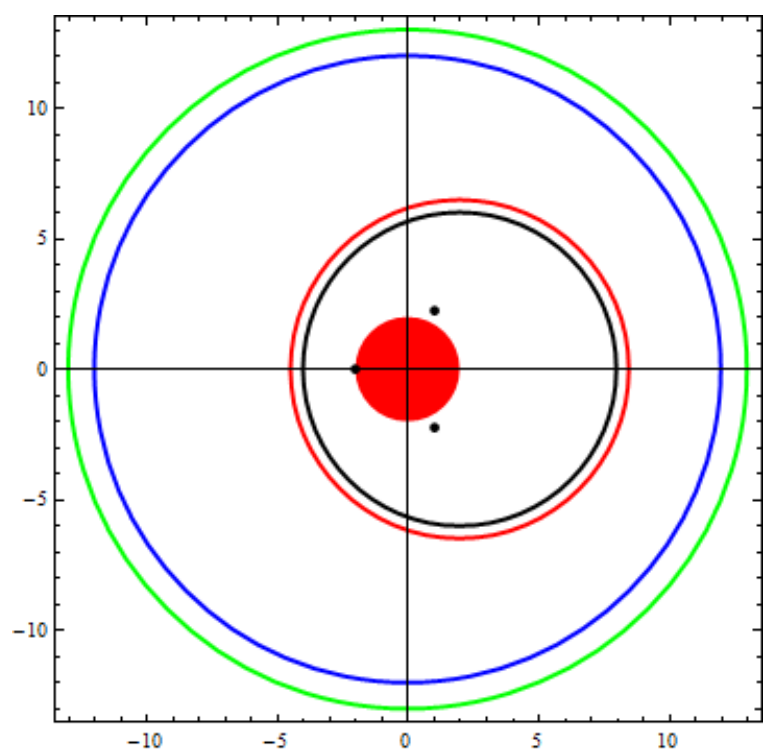

\section{Proof of Theorem}

Proof of Theorem 1. Consider the polynomial

$$
\begin{aligned}
F(z) & =(t-z) P(z)=(t-z)\left(a_{n} z^{n}+a_{\mu} z^{\mu}+\ldots+a_{1} z+a_{0}\right) \\
& =-a_{n} z^{n+1}+t a_{n} z^{n}-a_{\mu} z^{\mu+1}+\left(t a_{\mu}-a_{\mu-1}\right) z^{\mu} \\
& +\left(t a_{\mu-1}-a_{\mu-2}\right) z^{\mu-1}+\ldots+\left(t a_{1}-a_{0}\right) z+t a_{0} .
\end{aligned}
$$


This gives

$$
\begin{gathered}
|F(z)|=\mid-a_{n} z^{n+1}+t a_{n} z^{n}-a_{\mu} z^{\mu+1}+\left(t a_{\mu}-a_{\mu-1}\right) z^{\mu}+\left(t a_{\mu-1}-a_{\mu-2}\right) z^{\mu-1} \\
+\ldots+\left(t a_{1}-a_{0}\right) z+t a_{0}|\geq| a_{n}|| z^{n} \mid\left[|z-t|-\sum_{j=0}^{\mu+1}\left|\frac{t a_{j}-a_{j-1}}{a_{n}}\right| \frac{1}{|z|^{n-j}}\right] .
\end{gathered}
$$

Since $p>0, q>0$ and $p^{-1}+q^{-1}=1$, therefore we have by Hölder's inequality for $|z| \geq t$

$$
|F(z)| \geq\left|a_{n}\right|\left|z^{n}\right|\left[|z-t|-(\mu+2)^{\frac{1}{q}}\left\{\sum_{j=0}^{\mu+1}\left|\frac{t a_{j}-a_{j-1}}{a_{n} t^{n-j}}\right|^{p}\right\}^{\frac{1}{p}}\right]>0
$$

if

$$
|z-t|>(\mu+2)^{\frac{1}{q}}\left\{\sum_{j=0}^{\mu+1}\left|\frac{t a_{j}-a_{j-1}}{a_{n} t^{n-j}}\right|^{p}\right\}^{\frac{1}{p}} .
$$

This shows that for $|z| \geq t, F(z)$ does not vanish in

$$
|z-t|>(\mu+2)^{\frac{1}{q}}\left\{\sum_{j=0}^{\mu+1}\left|\frac{t a_{j}-a_{j-1}}{a_{n} t^{n-j}}\right|^{p}\right\}^{\frac{1}{p}} .
$$

Hence, we conclude that those zeros of $F(z)$ and therefore $P(z)$ whose modulus is greater than $t$ lie in

$$
|z-t| \leq(\mu+2)^{\frac{1}{q}}\left\{\sum_{j=0}^{\mu+1}\left|\frac{t a_{j}-a_{j-1}}{a_{n} t^{n-j}}\right|^{p}\right\}^{\frac{1}{p}} .
$$

This completes proof of Theorem 1.

\section{References}

[1] R.E. Allardice, On a limit of the roots of an equation that is independent of all but two of the coefficients, Bull. Amer. Math. Soc. 13 (1906-1907) 443-447.

[2] A. Aziz, N.A. Rather, Bounds for the zeros of a class of Lacunary-type polynomials, Journal of Mathematical Inequalities 73 (2013) 445-452.

[3] A.L. Cauchy, Exercise de mathematique, In Oeuvres 9 (2) (1829) 122.

[4] G. Eneström, Remargue sur an theoreme relatif aux racines de 1 equation $a_{n} x^{n}+$ $\cdots+a_{0}=0$ ou tousles coefficients sont reels et positifs, Tohoku Math. J. 18 (1920), 34-36, Translation of a Swedish article in Ofversigt of Konigl. Vetenskaps Akademiens Forhandlingar, 50 (1893) 405-415. 
[5] L. Fejér, Sur la racine de moindre module d'une equation algebrique, C. R. Acad. Sci. Paris 145 (1907) 459-461.

[6] L. Fejér, Über die Wurzel von kleinsten absoluten Beträge einer algebraischen Gleichung, Math. Ann. 65 (1908) 413-423.

[7] A. Hurwitz, Über die Nullslellen des Bessel'schen Function, Math. Ann. 33 (1889) 246-266.

[8] A. Joyal, G. Labelle, Q.I. Rahman, On the location of zeros of polynomials, Canad. Math. J. Bull. 10 (1967) 53-63.

[9] S. Kakeya, On the limits of the roots of an algebraic equation with positive coefficients, Tohoku Math. J. 2 (1912-13) 140-142.

[10] E. Landau, Über den Picardschen Satz, Vierteljahrsschrift Naturforsch. Gesellschaft Zürcher \& Furrer 51 (1906) 252-318.

[11] E. Landau, Sur quelques generalisations du theoreme de M. Picard, Ann. Ecole Norm. 24 (3) (1907) 179-201.

[12] M. Marden, Geometry of Polynomials, Math. Surveys No. 3, Amer. Math. Soc. Providence R. I., 1949.

[13] G.V. Milovanovic, D.S. Mitrinovic, Th.M. Rassias, Topics in Polynomials, Extermal Problems, Inequalities, Zeros, World Scientific, Singapore, 1994.

[14] E.B. Van Vleck, On the limits to the absolute values of the roots of a polynomial, Bull. Soc. Math. France 53 (1925) 105-125.

\section{DOI: $10.7862 /$ rf.2018.12}

\section{Idrees Qasim}

email: idreesf 3@gmail.com

ORCID: 0000-0001-5838-6522

Department of Mathematics

National Institute of Technology Srinagar - 190006

INDIA

\section{Tawheeda Rasool}

email: tawheedrasool@gmail.com

Department of Mathematics

National Institute of Technology Srinagar - 190006

INDIA 
Abdul Liman

email: abliman@rediffmail.com

Department of Mathematics

National Institute of Technology Srinagar - 190006

INDIA

Received 10.11.2017

Accepted 31.01.2018 